\title{
An addendum
}

\author{
By C. E. Walsh.
}

(Received 20th June 1934. Read 2nd November, 1934.)

A generalised lemma used in the second of two papers ${ }^{1}$ enables us, as was suggested there, to extend results of the first. Thus, among others, we easily get the following:

Let $t_{n}$ be determined by the relation

$$
\begin{gathered}
t_{n}=c_{n}^{1} t_{n-1}+c_{n}^{2} t_{n-2}+\ldots+c_{n}^{m} t_{n-m}+e_{n} y_{n} \\
\text { where lim } y_{n}=0 .
\end{gathered}
$$

Then $t_{n} \rightarrow 0$, if, denoting $\left|c_{n}^{r}\right|$ by $d_{n}^{r}$ everywhere,

(i) $d_{n}^{1}<1$ for all $n$

(ii) $\prod_{p=1}^{n} d_{p}^{1} \rightarrow 0$ as $n \rightarrow \infty$

(iii) $\left|e_{n}\right| \leqslant K\left(1-d_{n}^{1}\right)$, where $K$ is fixed

(iv) $\prod_{n=1}^{\infty}\left(1+\frac{d_{n}^{2}}{d_{n}^{1} d_{n-1}^{1}}+\frac{d_{n}^{3}}{d_{n}^{1} d_{n-1}^{1} d_{n-2}^{1}}+\ldots+\frac{d_{n}^{m}}{d_{n}^{1} \frac{1}{d_{n-1}^{1}} \ldots d_{n-m+1}^{1}}\right)$. is convergent.

Condition (iv) will be satisfied if the series

$$
\sum^{n=\infty} d_{n}^{1-} d_{n-1}^{1} \cdots \bar{d}_{n-r+1}^{1} \quad \text { converge, where } r=2,3, \ldots m .
$$

Making the substitutions

$$
c_{n}^{r}=\frac{a_{n}^{r}}{1+a_{n}^{1}}, \quad e_{n}=\frac{1}{1+a_{n}^{1}}
$$

a theorem recently proved by Sunouchi ${ }^{2}$ follows, namely :

Let $\left(1+a_{n}^{1}\right) t_{n}=a_{n}^{1} t_{n-1}+a_{n}^{2} t_{n-2}+\ldots+a_{n}^{m} t_{n-m}+y_{n}$ where $\lim y_{n}=0$.

Then $t_{n} \rightarrow 0$ if

(i) $a_{n}^{1}>0$ for all $n$,

(ii) $\sum_{n=1}^{\infty} \frac{1}{a_{n}^{\mathbf{1}}}$ diverges,

(iii) $\sum_{n=,}^{\infty}, \frac{a_{n}^{r} \mid{ }^{r}-1}{a_{n}^{1}} \prod_{s=1}^{1}\left(1+\frac{1}{a_{n-1}^{1}}\right)$ converges, where $r=2,3, \ldots m$.

${ }^{2}$ Proc. Edinburgh Hath. Soc., (2) 3 (1932), 147-150 and 220-222.

$\approx$ G. Sunouchi, "Theorems on limits of recurrent sequences," Proc. Imperial Academy, X (1934) 4-7. 